\title{
Development of a Strong Stream Ciphering Technique Using Non-linear Fuzzy Logic Selector
}

\author{
Ahmed M. Al-Naamany and Afaq Ahmad \\ Information Engineering Department \\ College of Engineering, Sultan Qaboos University \\ P. O. Box 33, Postal Code 123; Muscat, Sultanate of Oman \\ Tel.: (968) 515 327, (968) 515 328; Fax. (968) 513 416E-mails: \\ naamany@squ.edu.om; afaq@squ.edu.om
}

\begin{abstract}
This paper introduces a new methodology of producing Pseudo- Random Binary Sequence (PRBS) of high complexity while with lesser burden of the processing requirements. The key idea behind the implementation of the proposed methodology is the use of a nonlinear fuzzy logic selector. A set of Linear Feedback Shift Registers (LFSRs) of nominal but varying lengths is used to reduce the processing as well as hardware burdens. In the proposed scheme, the LFSRs can be loaded with different configurations according to changed characteristic polynomials and polynomial seeds. This facilitation is being arranged such that they are to be selected by fuzzy logic selector switches. Further, the inputs to the fuzzy logic selector are based on userdefined parameters.
\end{abstract}

Key words: stream ciphering, linear feedback shift register, pseudo-random binary sequence, fuzzy logic, crypto analysis

\section{INTRODUCTION}

Security systems today are built on increasingly strong cryptographic algorithms that foil pattern analysis attempts [1]. The use of pseudo-random processes to generate secret quantities can result in pseudo-security [2]. The leading cryptographic concept is Data Encryption Standard (DES). It is a computerized extension of the old traditional methods, where, substitution

\footnotetext{
The original version of this chapter was revised: The copyright line was incorrect. This has been corrected. The Erratum to this chapter is available at DOI: 10.1007/978-0-387-35618-1_37
} 
and transpositions are being applied in series. Another major class of cryptosystems, popular in military applications is stream ciphers [1] - [7]. Most practical stream-cipher designs are centred on Linear Feedback Shift Registers (LFSRs). Although the Pseudo- Random Binary Sequence (PRBSs) generated by LFSRs satisfy all the randomness criteria, but the Beriekamp-Massey algorithm may recover the unknown state of a simple LFSR, and its feedback connection, with just 2n known bits of the PRBS. So that, the need to generate a more "complex" sequence led to the idea of using multiple LFSR's and somehow mixing their generated sequences so that the ultimate complexity would be the product of the individual complexities of the sequences. At present, most practical stream cipher designs centred on the non-linear combination of LFSRs [8] - [15].

In view of above discussed issues in the field of cryptography it is the need of the day that a strong stream cipher keys must be designed and analyzed. This is actually the prime justification to carry out this work. The aim of our work is to propose such LFSR based stream ciphers designs, which have stronger keys. To make keys as strong as possible needs the analysis of PRBS used in the stream ciphers. This paper introduces a new methodology of achieving highly complexed PRBS while with lesser burden of the processing requirements. The key idea behind the implementation of the proposed methodology is the use of a non-linear fuzzy logic selector. A set of Linear Feedback Shift Registers (LFSRs) of nominal but varying lengths is used to reduce the processing as well as hardware burdens. In the proposed scheme, the LFSRs can be loaded with different configurations according to changed characteristic polynomials and polynomial seeds. This facilitation is being arranged such that they are to be selected by fuzzy logic selector switches. Further, the inputs to the fuzzy logic selector are based on user-defined parameters.

\section{STREAM CIPHERING}

The one-time pad inspired the technique of stream ciphering. A stream cipher has perfect security, with the obvious disadvantage that it need unlimited amount of keys. And, thus suggests to encrypt the plain text by adding "Pseudo-Random" Binary Sequence (PRBS) generated by a deterministic algorithm. To decrypt the cipher-text, the process of subtraction of the added sequence is to be carried out [2], [7], [10], [12]. Linear feedback shift register, most often used in hardware designs, is the basis of generating PRBS for the use of stream ciphers. A string of bits is stored in a string of memory cells, and a clock pulse can advance the bits one space in that string. The XOR of certain positions in the string is used to 
produce the new bit in the string for each clock pulse. It is possible to choose the positions in the string to XOR so that, as long as the memory cells are not initially loaded with all zero bits, the period of the sequence of bits produced by that XOR may go up to $2^{n}-1$, where $n$ is the number of cells in the string [8], [9]. Figure 1 illustrates an LFSR $(n=4)$ associated with the characteristic polynomial $1+x+x^{4}$. If initial state, the contents of $s_{1}, s_{2}, s_{3}$, and $s 4$ is 1000 then, the output (PRBS) will be 000111101011001 ... which them repeats. The characteristic polynomial of this LFSR is $1+x+x^{4}$, which is primitive. This is so in general, an n-stage LFSR has maximal period $2^{\text {n-1 }}$ if and only if its characteristic polynomial is primitive.

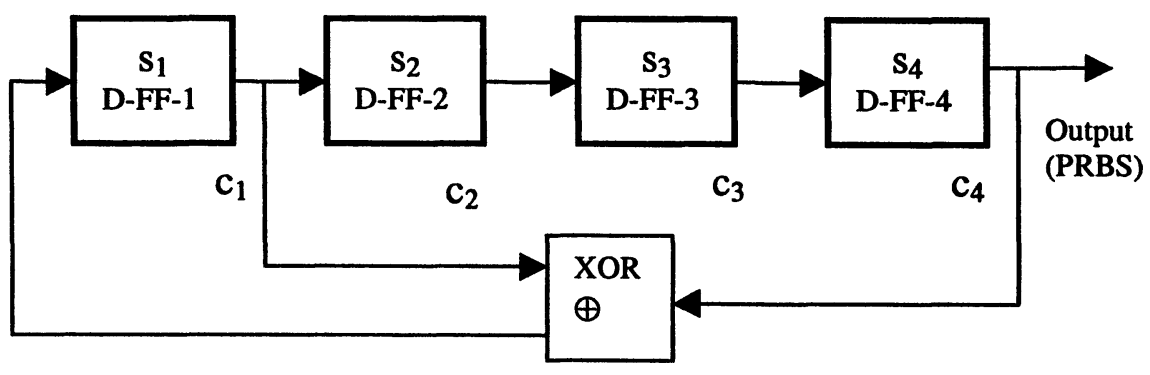

Figure 1: A 4-cell LFSR

Any such generated PRBS is ultimately periodic, and so closely related to cyclic codes. In application to cryptography some essential properties of the sequence should be required. These properties are: (i) period should be very large (say $10^{50}$ ), (ii) sequence should be easily generated through any deterministic algorithm, and (iii) Knowing a portion of plain text should not enable the cryptanalyst to reproduce the whole sequence. Unfortunately one can show (due to linearity) that $2 \mathrm{n}$ consecutive bits determine the whole sequence. Just 100 consecutive bits for the sequence of length $2^{50}-1$ gives the coefficients of the primitive polynomial hence the key LFSR. Using another or string of LFSRs to make the sequence nonlinear thereby strengthens the output from an LFSR. This technique can be applied in many ways like for the telecipher devices the use of LFSRs in pinwheels, Geffe generator [5] etc. Philosophy behind all such techniques is stem from the following fundamental facts: (i) choosing which combination or combinations of LFSRs is used to contribute to the output, (ii) being XORed with the output of the chosen LFSR or LFSR blocks, (iii) choosing the different characteristic polynomials of the LFSRs, and (iv) selecting a set of initial state of the LFSRs. Keeping in view of the above, for the first time we are exploring the feasibility of strengthening the output sequence by exploiting the technique of fuzzy logic. 


\section{FUZZY LOGIC SELECTOR - PROPOSED MODEL}

The fuzzy selector and the selected set of LFSR's each with different configurations are depicted in Figure 2. Figure 3, shows the details of fuzzy logic selector where, fuzzification transforms the crisp values of Mutual and Internal Keys into fuzzy sets. This transformation is governed by evaluating the membership functions represented in Figures 4 and 5 respectively. The fuzzy rule base contains the set of fuzzy rules in linguistic form, which is used to select a specific output from a given sets of input scenarios. The fuzzy rules used in this case are enumerated in Figure 6. The inference engine evaluates the rules and then based on the preconditions thus finally, recommends a set of actions at specific degree. The overall fuzzy output is the union of the outputs resulting from each rule. The defuzzification produces a non-fuzzy control action that represents the best of recommended fuzzy actions. The fuzzifier performs a mapping from a crisp point $\bar{x}=$ $\left(x_{1}, x_{n}\right)^{T} \in U$ ( universe of discourse) into a fuzzy set $A^{\prime}$ in $U$. We used the triangular shaped fuzzifier (the most commonly used). The membership function for each term in the partitioned spaced is expressed mathematically as of $[1,2]$ and given in Equation (1) below:

$$
\mu_{A}(x)=\left\{\begin{array}{cc}
0 & \left|x-x_{0}\right| \geq \alpha^{-1} \\
1-\left|x-x_{0}\right| \alpha & \text { otherwise }
\end{array}\right.
$$

Where $\mathbf{x}_{0}$ and $\mathbf{a}$ are the center and the slope of the triangular membership function respectively. In the case of this model the two variables considered; are the Mutual Key and Internal Built-In key whose each values are fed into the fuzzy selector to achieve the desired non-linear selection of the LFSR to be used. For simplicity each variable's universe of discourse is divided into three input membership functions, Zero, Middle and High. This is seen to be adequate as the inputs ranges from zero to 15 only. 


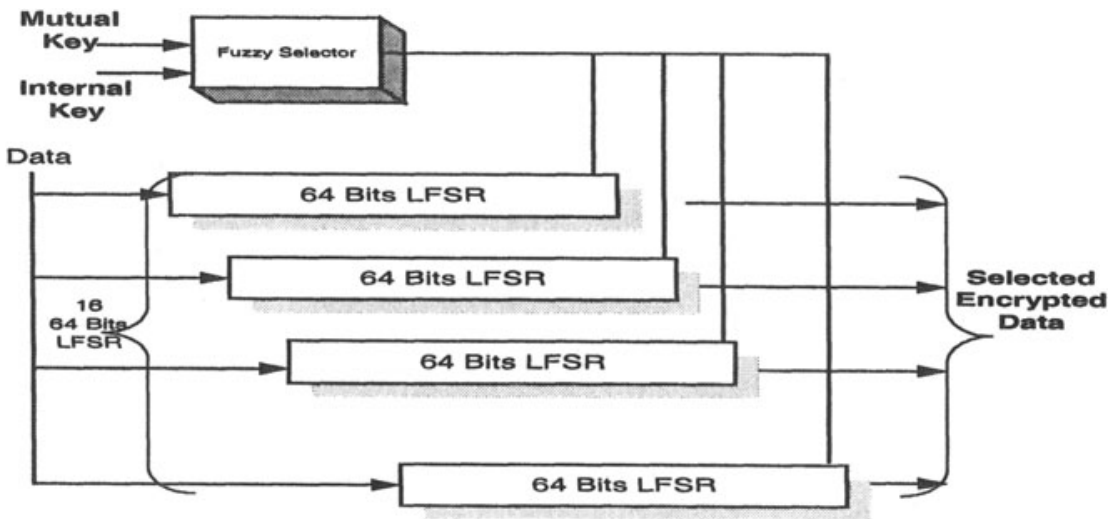

Figure 2: The Fuzzy Selector system with the LFSR's

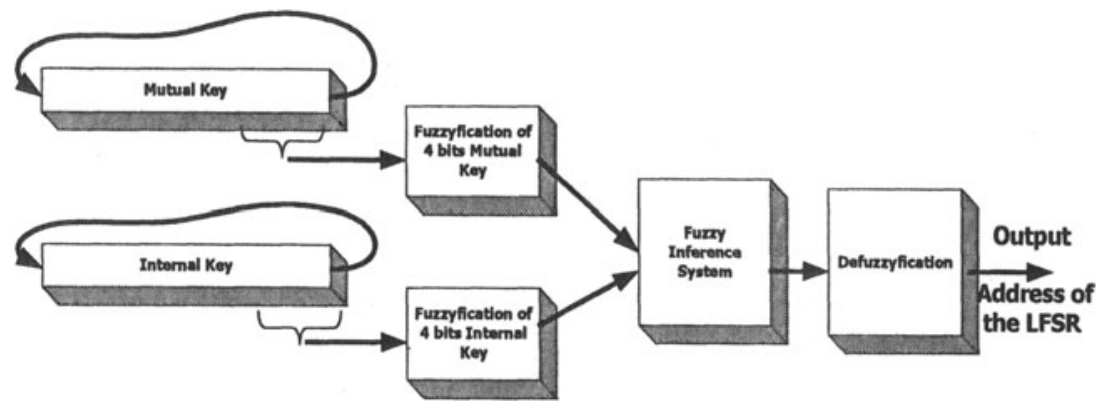

Figure 3: Details of Fuzzy Selector

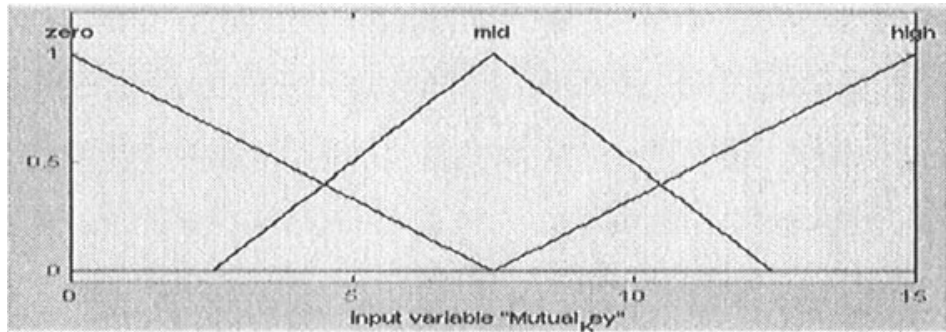

Figure 4: Fuzzification of Mutual Key

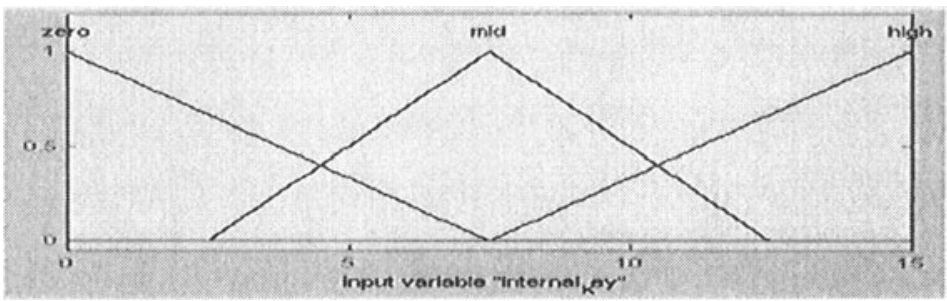

Figure 5: Fuzzification of Internal Key 


\section{IMPLEMENTATION CRITERIA}

\subsection{The Fuzzy Rule Base}

The rule base is set up using IF-THEN rules based on the fuzzy model. The IF-THEN rules describe what action is to be taken if a certain set of conditions is met. It incorporates information about every possible condition that the system can encounter. Note that when a fuzzy rule base recognizes information that is partially true it can partially invoke more than one rule at a time. If two or more rules are to be invoked that have the same value, than the rule that is invoked to the greatest degree is chosen (the maximum), and the rest are discarded [16], [17]. This is the union fuzzy operation. In our model of the system fuzzy logic rule base is represented as a two-dimensional table look up as shown in Figure 6.

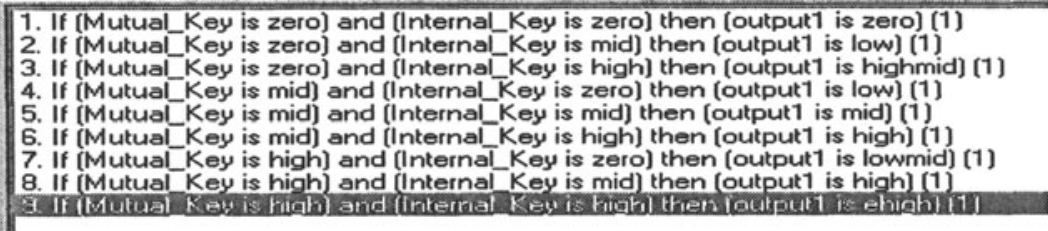

Figure 6: Fuzzy rule base of Fuzzy Inference System.

\subsection{The Fuzzy Inference Engine and Defuzzification}

In evaluating the rule we used the minimum operator (the fuzzy AND operator), i.e. $\mu^{\mathrm{i}} o^{i}=\mu_{\mathrm{I}_{1}^{\mathrm{i}}}\left(x_{1}\right) \wedge \mu_{\mathrm{I}_{2}}\left(x_{2}\right)$ where $O^{\mathrm{i}}$ denotes the output region of rule $\mathrm{i}$, and $I_{j}^{i}$ denotes the input region of Rule $\mathrm{i}$ for the $\mathrm{j}$ components, $x_{1}$ and $x_{2}$ are the two inputs, e and $e$ in our case. For the defuzzification an average (Centroid) defuzzification formula (Equation 2) is used to determine the outputs.

$$
y_{i}=\frac{\sum_{i=1}^{M} \mu^{i} o_{j}^{i} \bar{y}_{j}^{i}}{\sum_{i=1}^{M} \mu^{i}{ }^{i}{ }_{j}^{i}}
$$

Where $\mathrm{j}$ denotes the $\mathrm{jth}$ component of the output vector $\left(\mathrm{O}_{\mathrm{j}}^{\mathrm{i}}\right.$ is the region of Rule $\mathrm{i}$ for the jth output component, $\bar{y}_{j}^{i}$ denotes the center value of region $O^{i}$ $\mathrm{j}, \mathrm{M}$ is the number of fuzzy rules in the combined fuzzy rule base. Dividing it into nine memberships allowing specific selections to be chosen for every 
input configuration performs the output defuzzification. The memberships are depicted in Figure 7.

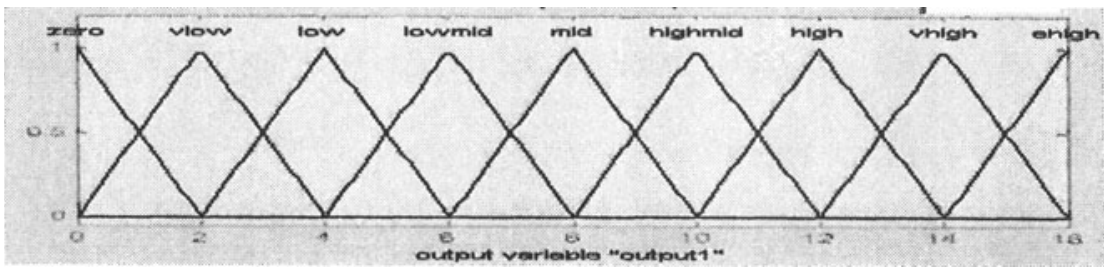

Figure 7: Defuzzification of output selection

\section{RESULTS \& CONCLUSIONS}

Figure 8 shows the result where output, internal and mutual keys can be clearly related with each other. Through this paper we put forward an approach of generating PRBS of high complexity with nominal overhead of processing cost and time. We have compared our approach with other existing methodologies for the achievement of same objective. It has been noted that this method is capable of giving very much justifiable positive indicator towards the direction of feasibility of using the Fuzzy logic theory to achieve the more complexed sequences for the use of stream ciphering.

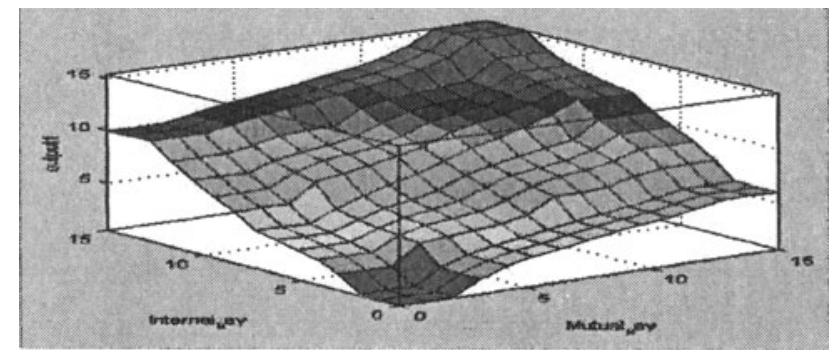

Figure 8: Output and keys selection

\section{REFERENCES}

[1]. Jovan $D_{\mathrm{j}}$. Golic, 'Recent advances in cryptanalysis', publications de 1 'InstitutMathematique, vol.64/78, pp.183-204, 1998

[2]. Johansson, T., 'Collation Attacks On Stream Ciphers And Related Decoding Problems', Information Theory Workshop, pp.156-157, 1998

[3]. Blackburn, S.R., Brincat, K., Mirza, F. and Murphy, S., 'Cryptanalysis of Stream Cipher', Electronic Letters, Vol. 34 no. 12, pp. 1220-1221, 11 June 1998. 
[4]. Chi-Chun Lo, and Yu-Jen Chen, 'Secure Communication Mechanisms For GSM Networks', IEEE Transaction On Consumer Electronics, vol. 45 no. 4, pp. 1074-1080, 1999.

[5]. Geffe P.R, 'How to protect data with Ciphers that are really hard to break', Electronics, pp. 99-101, Jan. 4, 1973

[6]. Alan G. Konheim, 'Cryptography: A Primer,' A Wiley-Inter-science Publication, John Wiley \& Sons, 1981

[7]. Chi-Kwong Chan and L.M. Cheng, 'Design of key stream generator Electronics Letters IEE, June 1998, vol.34, no.12, pp 1206-07, 1998

[8]. Solomon W. Golomb, 'Shift Register Sequences', Aegean Park Press Revised Edition 1982

[9]. Wayne G. Barker, 'Cryptanalysis of Shift-Register Generated Stream Cipher Systems', Aegean Park Press, 1984

[10]. Zeng, K., Yang, C. -H., Wei, D. -Y. and Rao, T.R.N., 'Generators In Stream - Cipher Cryptography', Computer, vol 24 no. 2 pp. 8-17, Feb. 1991

[11]. Chi-Kwong Chan, Cheng, L.M., 'The CHNN Non-linear Combination Generator', IEEE International Conference On Electronics, Circuits and Systems, vol.2, pp.257-260, 1998

[12]. Dachselt, F., Kelber, K., Schwarz, W. and Vandewalle, J., 'Chaotic Versus Classical Stream Ciphers- A Comparative Study', IEEE International Symposium on Circuits And Systems vol.4, pp.518521,1998

[13]. Chaoping Xing, and Kwok Yan Lam, 'Sequences With Almost Perfect Linear Complexity Profiles And Curves Over Finite Fields', IEEE Transactions On Information Theory, vol. 45 no. 4, pp. 1267-1270, 1999

[14]. J. $D_{j}$. Golic, 'Cryptanalysis of Three Mutually Clock-Controlled Stop/Go Shift Registers', IEEE Transactions on Information Technology, Vol. 46, No. 3, pp 1081-1090, May 2000.

[15]. Ahmad A., Al-Mushrafi M. J, Al-Busaidi S., Al-Naamany A., Jervase J., 'An NLFSR Based Sequences Generator for Stream Ciphers', SETA01 conf. pp. 11-12 May 13-17, 2001 Bergen

[16]. Wang, L. ; "Adaptive fuzzy systems and control, design and stability analysis"; 1994, Prentice Hall.

[17]. J. Jang, "Neuro-Fuzzy and Soft Computing" Prentice Hall, New Jersey, USA, 1997 